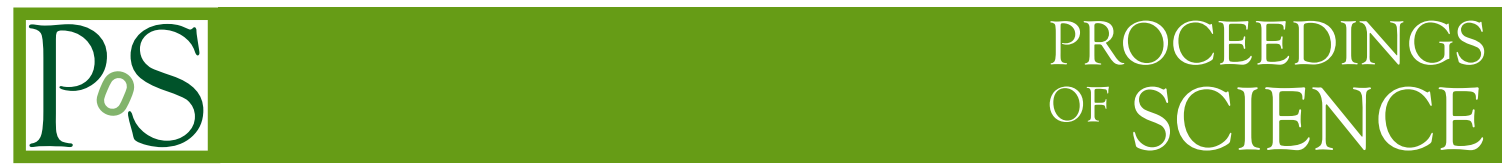

\title{
Direct Detection of Dark Matter
}

\author{
Hyun Su Lee* \\ Center for Underground Physics, Institute for Basic Science (IBS), Daejeon 34126, Republic of \\ Korea \\ E-mail: hyunsulee@ibs.re.kr
}

This article is a brief overview of the status of direct detection experiments of the dark matter, especially searching for WIMPs (Weakly Interacting Massive Particles) at deep underground laboratory, when it was discussed at 37th international conference on high energy physics (ICHEP2018) in Seoul, Korea.

The 39th International Conference on High Energy Physics (ICHEP2018)

4-11 July, 2018

Seoul, Korea

${ }^{*}$ Speaker. 


\section{Introduction}

There is compelling evidences for the dominant matter component of the universe is not made of ordinary matter, but rather non-baryonic dark matter [1,2,3]. Cosmological observations tell us that approximately $26 \%$ of the mass and energy in the universe is the dark matter while ordinary matter is only approximatly $5 \%[4,5,6,7,8]$. The remained $69 \%$ is homogeneous dark energy. Although the underlying nature of dark matter remains elusive, there are a number of particle dark matter candidates $[9,10]$ that describe the observed structure of the universe as one can see in Fig. 1. One of the most stringent candidate is super-symmetric WIMPs (Weakly Interacting Massive Particles) [11, 12, 13].

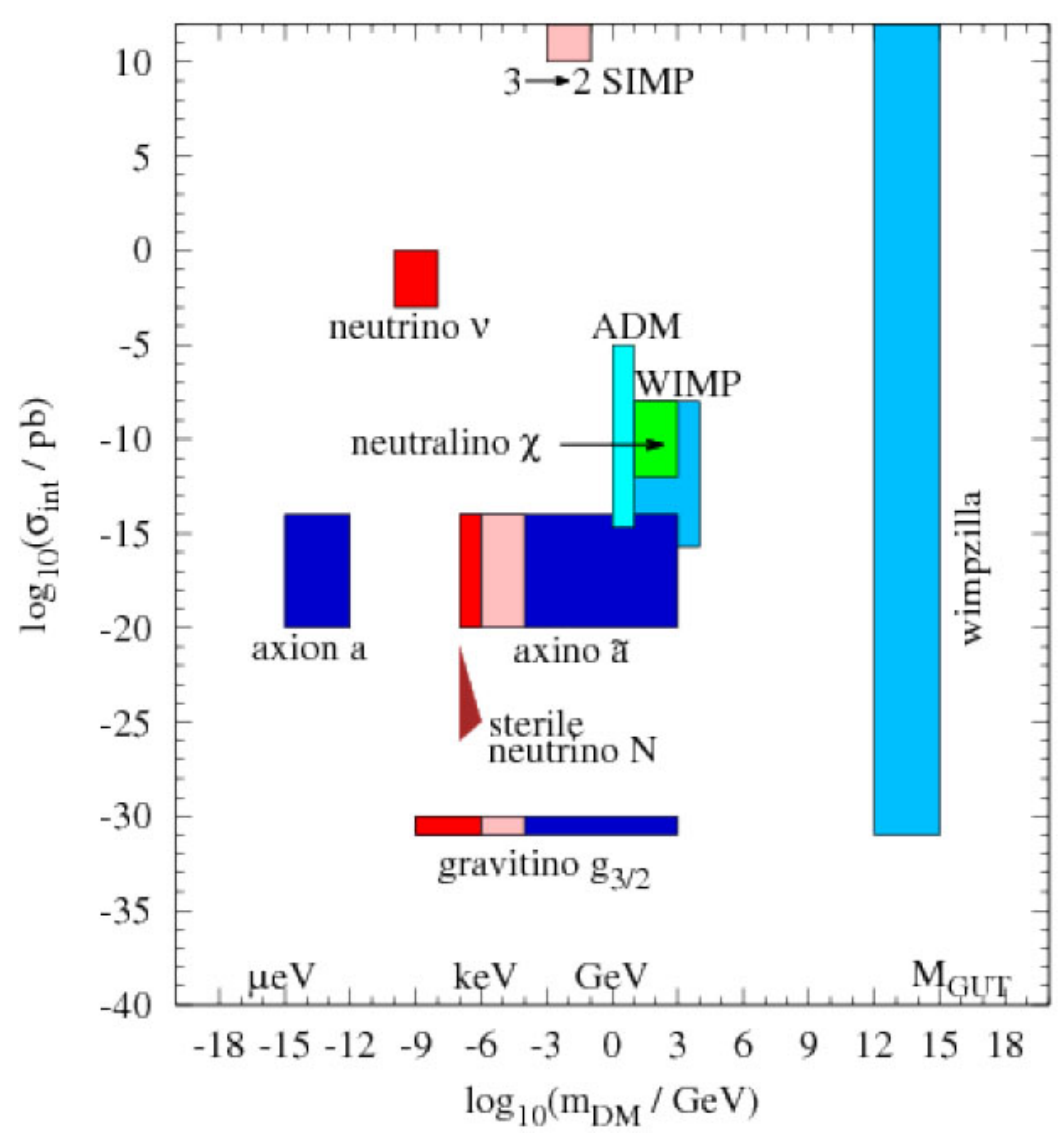

Figure 1: A variety of well motivated particle dark matter candidates are shown in the log-log plane of dark matter mass and interaction cross section. This plot is taken from Ref. [10]

WIMP dark matter has been searched in three different ways consisting of direct $[14,15,16]$, indirect [17, 18, 19], and collider production [20, 21] with no clear success [22]. Direct searches of the WIMP dark matter in deep underground laboratory have been performed by searching for the scattering process of the WIMP dark matter with ordinary matter, which deposits tiny energy in target material scattered off nuclei [12, 23, 24]. In this article, we present current status of direct detection experiments for WIMP dark matter. 


\section{Current status of direct detection}

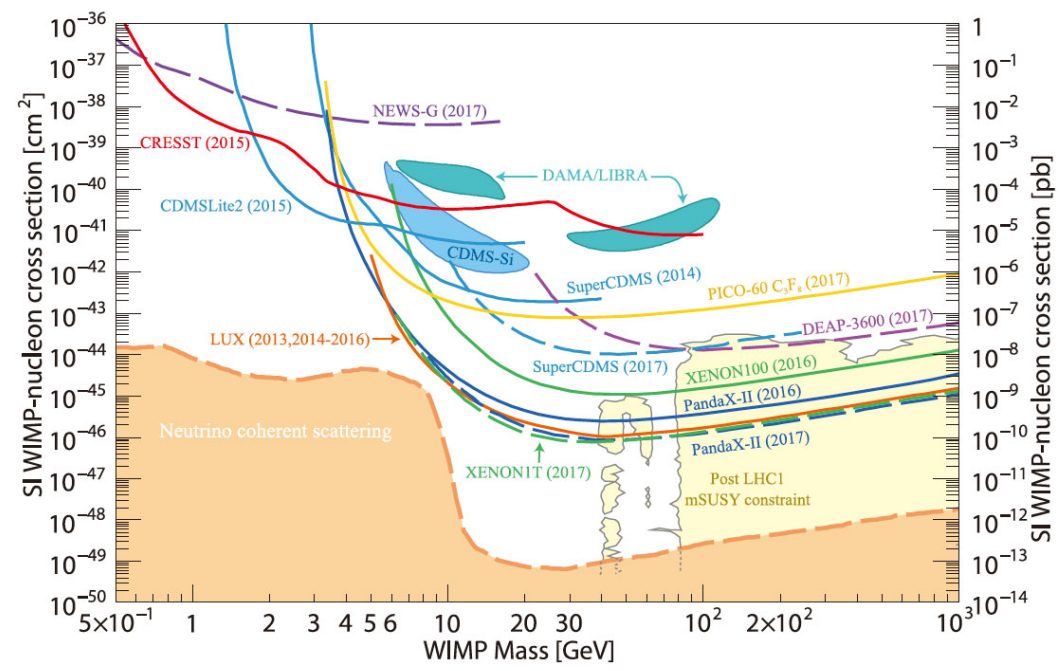

Figure 2: Limits on WIMP-nucleon cross-section for spin-independent coupling as a function of WIMP masses are presented. This plot is taken from Ref. [22]

Figure 2 shows the current status of direct detection experiments as limits on WIMP-nucleon cross-section for spin-independent interaction as a function of WIMP masses taken from recent particle data group reports [22]. In summary, liquid noble gas detectors have achieved big progress in sensitivities for the high-mass WIMP searches while many different types of detectors have developed and competed for the low-mass dark matter searches. However, a long debated annual modulation signals observed by DAMA collaboration has still been unresolved.

\section{High mass searches}

Liquid noble gas (Xe, Ar) dual-phase detectors have lead WIMP dark matter searching for the mass greater than $10 \mathrm{GeV} / \mathrm{c}^{2}$ (high-mass) last decade with tremendous progress. Liquid and gas two-phase detectors allows one to measure both the primary scintillation and the secondary ioniztion electrons drifted through the liquid, amplified in the gas stage. The ratio of the primary and secondary signal allow one to discriminate the electron recoil backgrounds from the nuclear recoil signals together with 3-dimensional position reconstruction. This makes the liquid noble gas detectors to search WIMP dark matter with almost background-free conditions in the region of interest. Due to their relatively easy scalability as well as high radiopurity, which has been achieved with extensive works, this type detectors improved detection sensitivities of about three order of magnitudes during last 10 years for high-mass WIMP searches.

XENON collaboration progresses quite fastly and leads high-mass search starting from XENON10 [25] operated at Gransasso Underground Laboratory since 2006. Its sucessors have continued with XENON100 [26] and XENON1T [16]. Current best limit for high-mass dark matter searches is obtained from XENON1T with 1 ton-year data exposure obtaining 90\% confidence level (CL) upper limit of $4.1 \times 10^{-47} \mathrm{~cm}^{2}$ for WIMP-nucleon spin-independent cross section in case of WIMP 
mass $30 \mathrm{GeV} / \mathrm{c}^{2}$. Fast upgrade for XENONnT is planned at 2019 with replacement of inner liquid Xenon vessel to accomodate 8-ton liquid Xenon. LZ (Lux-Zeplin), an upgrade of LUX experiment that had an operation since 2014 with a $307 \mathrm{~kg}$ liquid xenon at Standford Underground Research Facility [27], has well prepared to start operation at 2020 spring with 10-ton liquid xenon. With successful operation of PandaX at Jingping Underground Laboratory [28], the collaboration has a plan to upgrade detector as PandaX-4T at 2019-2020.

Liquid Ar experiments have two different approaches of single-phase by DEAP-3600 experiment [29] at SNO Underground Laboratory and two-phase by DarkSide-50 [30] at LNGS (Granssaso) with similar performance. The community of liquid Ar experiments agrees to launch joint collaboration for single future experiment of the DarkSide-20k with 50 ton of underground liquid Ar. It will eventually be upgraded to 300 ton detector. Figure 3 show projected sensitivities of liquid noble gas experiments that are explained here.

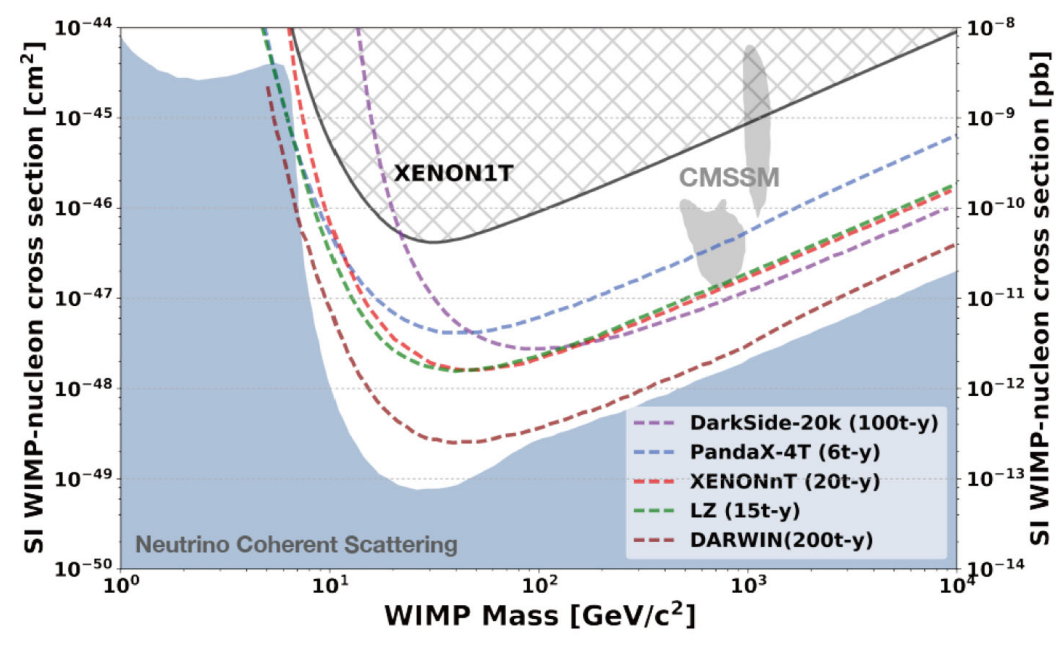

Figure 3: Projected sensitivities of planned liquid noble gas experiments. This plot is taken from Ref. [31]

\section{Low mass searches}

Various techniques are developed to reach enough sensitivities for low-mass dark matter between few hundreds $\mathrm{MeV} / \mathrm{c}^{2}$ and sub GeV/c ${ }^{2}$. DarkSide-50 produce interesting results by using so called "s2" only analysis [32]. Only using ionization signals, which are amplified in gas stage, makes low energy threshold of $0.1 \mathrm{keV}_{e e}$ (electron-equivalent energy). With this approach, they obtain the best exclusion limit for the WIMP dark matter in the mass range of $1.8-6 \mathrm{GeV} / \mathrm{c}^{2}$.

The CRESST-III experiment upgrade their detector module consisted to a $24 \mathrm{~g} \mathrm{CaWO}_{4}$ crystal that is connected to transition-edge-sensors for signal readout of phonons [33]. A silicon-onsapphire disc is used for a light absorber. The mass of CRESST-III is significantly lower than that of CRESST-II about $300 \mathrm{~g}$ [34]. Main goal is to achieve lower energy threshold for lower mass dark matter search. The CRESST-III achieved less than $100 \mathrm{eV}$ energy threshold while CRESST-II was $307 \mathrm{eV}$. Results from single module for $2.39 \mathrm{~kg}$.days exposure already obtain compatible limits 
with CRESST-II $52 \mathrm{~kg}$. days exposure for WIMP mass less than $20 \mathrm{GeV} / \mathrm{c}^{2}$ and much better results for WIMP mass below $1 \mathrm{GeV} / \mathrm{c}^{2}$.

The CDEX experiment has developed p-type point-contact germanium detector to reach lowenergy threshold. The CDEX-10 experiment with a $10 \mathrm{~kg}$ germanium detector array immersed in liquid nitrogen at the China Jinping Underground Laboratory can reach to $160 \mathrm{eV}_{e e}$ analysis threshold improving the exclusion limit significantly [35]. The CDEX has plan for upgrade detectors up to 1-ton scale that may be grown in the Jinping Underground Laboratory for low background.

The NEWS-G experiment has developed gases spherical proportional detector with light noble gases to search for low-mass WIMP. A $60 \mathrm{~cm}$ diameter prototype detector operated with a mixture of $\mathrm{Ne}$ and $\mathrm{CH}_{4}$ at 3.1 bars for a total exposure of $9.6 \mathrm{~kg}$.days [36]. Achieved low energy threshold of $150 \mathrm{eV}_{e e}$ allows the best limit for WIMP mass around $0.5 \mathrm{GeV} / \mathrm{c}^{2}$.

The observed upper limits from various low-mass dark matter serach experiments are shown in Fig. 4.

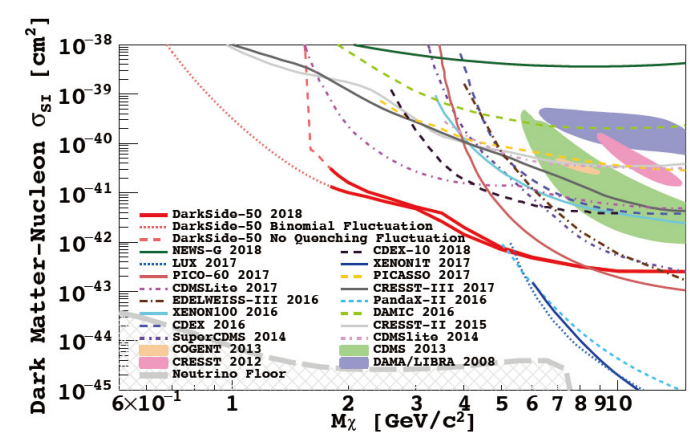

(a) DarkSide-50 [32]

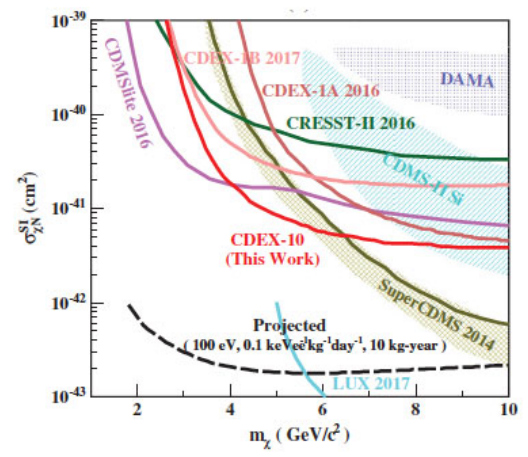

(c) CDEX-10 [35]

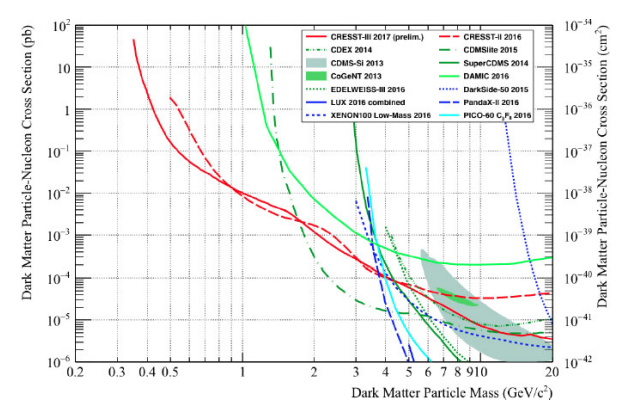

(b) CRESST-III [33]

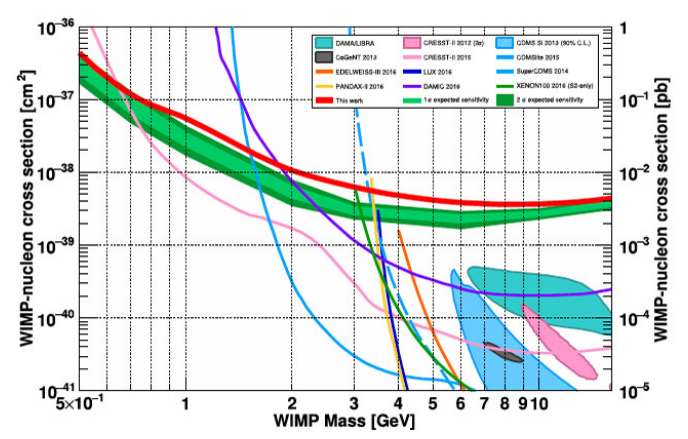

(d) NEWS-G [36]

Figure 4: Measured 90\% CL upper limits on the WIMP-nucleon spin-independent cross sections from various low-mass dark matter search experiments. Plots are taken from each reference.

\section{Tests of the DAMA annual modulation signal}

It has been a long time since DAMA reported an annual modulation in their event rate first time at 1998 [37] with an array of low-background $\mathrm{NaI}(\mathrm{Tl})$ crystals. Experiment has been upgraded and performed with much longer period of exposure and keeps reports about similar positive seasonal 
modulation [38, 39, 40]. The most recent result from DAMA/LIBRA-phase2 [41] shows a consistent annual modulation with more than $13 \sigma$ statistical significance as one can see in Fig. 5. Even though DAMA/LIBRA-phase2 data itself is inconsistent with canonical spin-independent WIMP dark matter model $[42,43]$ in the context of standard halo model, but it still has consistent phase and period with previous DAMA/NaI and DAMA/LIBRA-phase1 results.

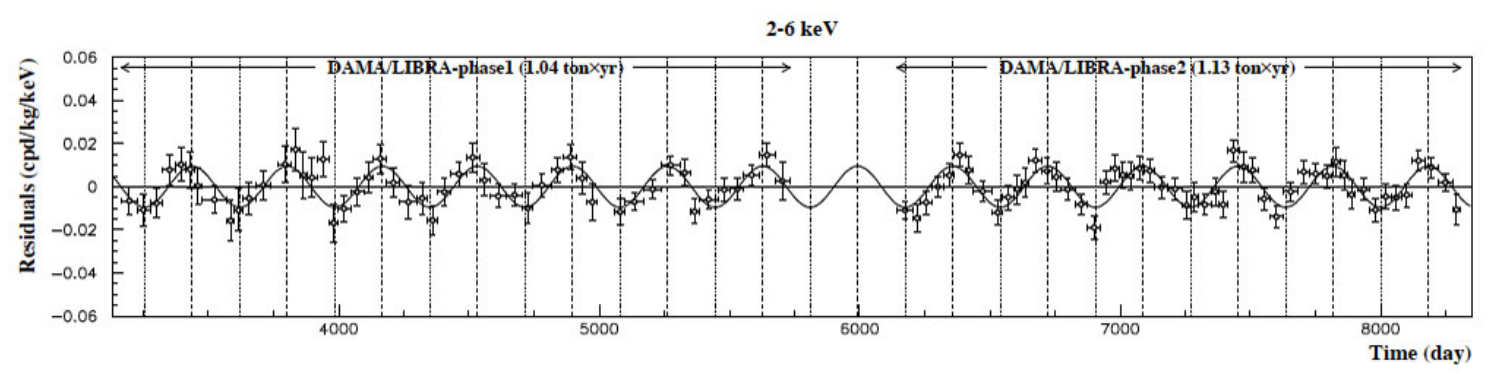

Figure 5: Observed annual modulation of event rates (residual spectrum) measured by DAMA/LIBRAphase 1 and phase 2 in 2-6 keV energy region. This plot is taken from Ref. [41]

If the annual modulation signal observed by DAMA is interpreted in the context of the standard halo WIMP dark matter model, i.e. interpretation of DAMA/LIBRA-phase1 result [44], the favored regions of WIMP-nucleon cross section have been already excluded by other direct search experiments $[22,16]$. It is also been studied for the annual modulation of event rates by other experimental searches with liquid Xenon [45, 46, 47], but they clearly found contradictory results with DAMA. Therefore, it is important to devise experiments that search for the DAMA signal using the same technique of $\mathrm{NaI}(\mathrm{Tl})$ crystals with higher sensitivity.

To reproduce the DAMA observation, several experiments [48, 49, 50, 51, 52, 53] have attempt to search WIMP dark matter using the same NaI(Tl) crystals. ANAIS [54] and COSINE [50, 55] experiments start physics data taking with approximately $100 \mathrm{~kg}$ scale detectors. Interesting first result from COSINE-100 with 59.5 days data indicate that DAMA's annual modulation signal is in tension with typical dark matter interpretation of spin-independent interaction with the standard halo model based on their precise background understanding [56]. This is consistent with all other null results experiments but, it is first time with same $\mathrm{NaI}(\mathrm{Tl})$ detector. It will be more interesting to compare the modulation amplitudes observed by the ANAIS and COSINE experiments that will be available in near future.

Even though ANAIS and COSINE take physics data, crystal backgrounds achieved by those experiments [55, 57, 58, 59, 60] are still higher than those of the DAMA/LIBRA's crystals [41]. It has potential leak to resolve all possible scenarios of the DAMA's modulation signals due to poorer detector performances. COSINE, SABRE, and PICO-LON experiments independently have developed ultra-low background $\mathrm{NaI}(\mathrm{Tl})$ detectors with a goal of lower background level than DAMA/LIBRA's crystals. Each experiment has proper progress of crystal growing. It is expected to have conclusive understanding of the DAMA's signal within a few years.

\section{Summary}

The WIMP dark matter direct searches in deep underground are actively ongoing with 
significant progress last few decades even though we did not find crucial evidence of the WIMP dark matter. In high-mass region, liquid Xe experiments made leading role in the field. Near future project are well prepared in liquid Xe and liquid Ar with significant improvement on the sensitivities of the search regions. In the low-mass region that requires a lower threshold, several new techniques are being developed to reach extremely low energy threshold. The DAMA conumdrum is still persistent but, new $\mathrm{NaI}(\mathrm{Tl})$ experiments will resolve this issue in near future.

\section{References}

[1] D. Clowe, A. Gonzalez, and M. Markevitch, Weak-lensing mass reconstruction of the interacting cluster 1E 0657-558: direct evidence for the existence of Dark Matter, Astrophys. J. 604 (2004) 596.

[2] D. Clowe et al., A direct empirical proof of the existence of Dark Matter, Astrophys. J. 648 (2006) L109.

[3] M. Persic, P. Salucci, and F. Stel, The universal rotation curve of spiral galaxies - I. The Dark Matter connection, Mon. Not. Roy. Astron. Soc. 281 (1996) 27.

[4] D. Larson et al., Seven-year Wilkinson microwave anisotropy probe (WMAP) observation: Power spectra and WMAP-derived parameters, Astrophys. J. Suppl. 192 (2011) 16.

[5] E. Komatsu et al., Seven-year Wilkinson microwave anisotropy probe (WMAP) observation: Cosmological interpretation, Astrophys. J. Suppl. 192 (2011) 18.

[6] M. Betoule et al. (SDSS Collaboration), Improved cosmological constraints from a joint analysis of the SDSS-II and SNLS supernova samples, Astron. Astrophys. 568 (2014) A22.

[7] P. A. R. Ade et al. (Planck Collaboraion), Plank 2015 results. XIII. Cosmological parameters, Astron. Astrophys. 571 (2016) A13.

[8] P. A. R. Ade et al. (Planck Collaboraion), Plank 2018 results. VI. Cosmological parameters, arXiv:1807.06209 (2018).

[9] G. Bertone, D. Hooper, and J. Silk, Particle dark matter: evidence, candidates and constraints, Phys. Rep. 405 (2005) 279.

[10] H. Baer, K.Y. Choi, J.E. Kim, and L. Roszkowski, Dark matter production in the early Universe: beyond the thermal WIMP paradiam, Phys. Rep. 555 (2015) 1.

[11] B.W. Lee and S. Weinberg, Cosmological Lower Bound on Heavy Neutrino Masses, Phys. Rev. Lett. 39 (1977) 165.

[12] M.W. Goodman and E. Witten, Detectability of Certain Dark Matter Candidates, Phys. Rev. D 31 (1985) 3059.

[13] G. Jungman, M. Kamionkowski, and K. Griest, Supersymmetric Dark Matter, Phys. Rep. 267 (1996) 195.

[14] R. Gaitskell, Direct Detection of Dark Matter, Ann. Rev. Nucl. Part. Sci. 54 (2004) 315.

[15] M. Battaglieri et al., US Cosmic Visions: New Ideas in Dark Matter 2017: Community Report, arXiv:1707.04591 (2017).

[16] E. Aprile et al. (XENON1T Collaboration), Dark Matter Search Results from a One Tonne $\times$ Year Exposure of XENONIT, Phys. Rev. Lett. 121 (2018) 111302.

[17] K. Choi et al. (Super-Kamiokande Collaboration), Search for neutrinos from annihilation of captured low-mass dark matter particles in the Sun by Super-Kamiokande, Phys. Rev. Lett. 114 (2015) 141301. 
[18] M.G. Aartsen et al. (IceCube Collaboration), Search for annihilating dark matter in the Sun with 3 years of IceCube data, Eur. Phys. J. C 77 (2017) 146.

[19] J. Conrad and O. Reimer, Indirect dark matter searches in gamma and cosmic rays, Nature Phys. 13 (2017) 224.

[20] M. Aaboud et al. (ATLAS Collaboration), Search for dark matter at $\sqrt{s}=13 \mathrm{TeV}$ in final states containing an energetic photon and large missing transverse momentum with the ATLAS detector, Eur. Phys. J. C 77 (2017) 393.

[21] A.M. Sirunyan et al. (CMS Collaboration), Search for dark matter in events with energetic, hadronically decaying top quarks and missing transverse momentum at $\sqrt{s}=13 \mathrm{TeV}$, JHEP 06 (2018) 027.

[22] M. Tanabashi et al. (Particle Data Group), The Review of Particle Physics, Phys. Rev. D 98 (2018) 030001.

[23] A.K. Drukier, K. Freese, and D.N. Spergel, Detecting cold dark-matter candidates, Phys. Rev. D 33 (1986) 3495.

[24] J.D. Lewin and R.F. Smith, Review of mathematics, numerical factors, and corrections for dark matter experiments based on elastic nuclear recoil, Astropart. Phys. 6 (1996) 87.

[25] J. Angle (XENON Collaboration), First Results from the XENON10 Dark Matter Experiment at the Gran Sasso National Laboratory, Phys. Rev. Lett. 100 (2008) 021303.

[26] E. Aprile (XENON Collaboration), First Dark Matter Results from the XENON100 Experiment, Phys. Rev. Lett. 105 (2010) 131302.

[27] D.S. Akerib (LUX Collaboration), Results from a search for dark matter in the complete LUX exposure, Phys. Rev. Lett. 118 (2017) 021303.

[28] X. Cui (PandaX-II Collaboration), Dark Matter Results from 54-Ton-Day Exposure of PandaX-II Experiment, Phys. Rev. Lett. 119 (2017) 181302.

[29] P.A. Amaudruz et al. (DEAP-3600 Collaboration), First results from the DEAP-3600 dark matter search with argon at SNOLAB, Phys. Rev. Lett. 121 (2018) 071801.

[30] P. Agness et al. (DarkSide Collaboration), DarkSide-50 532-day Dark Matter Search with Low-Radioactivity Argon, arXiv:1802.07198 (2018).

[31] K. Ni, Recent Results from Dark Matter Direct Detection Experiments, Presented in Thirteenth Conference on the Interactions of Particle and Nuclear Physics (CIPANP2018), May/28-Jun/3 (2018).

[32] P. Agness et al. (DarkSide Collaboration), Low-Mass Dark Matter Search with the DarkSide-50 Experiment, Phys. Rev. Lett. 121 (2018) 081307.

[33] F. Petricca et al. (CRESST Collaboration), First results on low-mass dark matter from the CRESST-III experiment, arXiv:1711.07692 (2017).

[34] G. Angloher et al. (CRESST Collaboration), Results on light dark matter particles with a low-threshold CRESST-II detector, Eur. Phys. J. C 76 (2016) 25.

[35] H. Jiang et al. (CDEX Collaboration), Limits on Light Weakly Interacting Massive Particles from the First $102.8 \mathrm{~kg} \times$ day Data of the CDEX-10 Experiment, Phys. Rev. Lett. 120 (2018) 241301.

[36] Q. Arnaud et al., First results from the NEWS-G direct dark matter search experiment at the LSM, Astropart. Phys. 97 (2018) 54. 
[37] R. Bernabei et al. (DAMA Collaboration), Searching for WIMPs by the annual modulation signature, Phys. Lett. B 424 (1998) 194.

[38] R. Bernabei et al. (DAMA/LIBRA Collaboration), First results from DAMA/LIBRA and the combined results with DAMA/NaI, Eur. Phys. J. C 56 (2008) 333.

[39] R. Bernabei et al. (DAMA/LIBRA Collaboration), New result of DAMA/LIBRA, Eur. Phys. J. C 67 (2010) 39.

[40] R. Bernabei et al. (DAMA/LIBRA Collaboration), Final model independent result of DAMA/LIBRA-phase1, Eur. Phys. J. C 73 (2013) 2648.

[41] R. Bernabei et al. (DAMA/LIBRA Collaboration), First model independent results from DAMA/LIBRA-phase2, arXiv:1805.10486 (2018).

[42] S. Baum, K. Freese, and C. Kelso, Dark Matter implications of DAMA/LIBRA-phase2 results, arXiv:1804.01231 (2018).

[43] S. Kang. S. Scopel, G. Tomar, and J.H. Yoon, DAMA/LIBRA-phase2 in WIMP effective models, JCAP 1807 (2018) 016.

[44] C. Savage, G. Gelmini, P. Gondolo, and K. Freese, Compatibility of DAMA/LIBRA dark matter detection with other searches, JCAP 0904 (2009) 010.

[45] E. Aprile (XENON Collaboration), Search for Electronic Recoil Event Rate Modulation with 4 Years of XENON100 Data, Phys. Rev. Lett. 118 (2017) 101101.

[46] K. Abe (XMASS Collaboration), Direct dark matter search by annual modulation with 2.7 years of XMASS-I data, Phys. Rev. D 97 (2018) 102006.

[47] D.S. Akerib (LUX Collaboration), Search for annual and diurnal rate modulations in the LUX experiment, Phys. Rev. D 98 (2018) 062005.

[48] J. Amare et al. (ANAIS Collaboration), From ANAIS-25 towards ANAIS-250, Phys. Procedia 61 (2015) 157.

[49] P. Adhikari et al. (KIMS Collaboration), Understanding internal backgrounds in NaI(Tl) crystals toward a $200 \mathrm{~kg}$ array for the KIMS-NaI experiment, Eur. Phys. J. C 76 (2016) 185.

[50] G. Adhikari et al. (COSINE-100 Collaboration), Initial Performance of the COSINE-100 Experiment, Eur. Phys. J. C 78 (2018) 107.

[51] K. Fushimi et al., Results from the first cryogenic NaI detector for the COSINUS project, JINST 12 (2017) P11007.

[52] C. Tomei et al. (SABRE Collaboration), SABRE: Dark matter annual modulation detection in the northern and southern hemisphere, Nucl. nstrum. Meth. A 845 (2017) 418.

[53] G. Angloher et al., Results from the first cryogenic NaI detector for the COSINUS project, JINST 12 (2017) P11007.

[54] J. Amare et al., The ANAIS-112 experiment at the Canfranc Underground Laboratory, arXiv:1710.03837 (2017).

[55] P. Adhikari et al. (COSINE-100 Collaboration), Background model for the NaI(Tl) crystals in COSINE-100, Eur. Phys. J. C 78 (2018) 490.

[56] C. Ha, Recent results in the COSINE-100 experiment, Presented in the 39th International Conference on High Energy Physics (ICHEP2018), 4-11 July, 2018. 
[57] P. Adhikari et al., Understanding internal backgrounds in NaI (Tl) crystals toward a $200 \mathrm{~kg}$ array for the KIMS-NaI experiment, Eur. Phys. J. C 76 (2016) 185.

[58] G. Adhikari et al., Understanding NaI(Tl) crystal background for dark matter searches, Eur. Phys. J. C 77 (2017) 437.

[59] J. Amare et al., Cosmogenic radionuclide production in NaI(Tl) crystals, JCAP 1502 (2015) 046.

[60] J. Amare et al., Assessment of backgrounds of the ANAIS experiment for dark matter direct detection, Eur. Phys. J. C 76 (2016) 1. 